\title{
Perspectives for Higgs measurements at Future Circular Collider
}

\author{
Ang LII ${ }^{a, 1, *}$ \\ ${ }^{a}$ Université de Paris, CNRS, Astroparticule et Cosmologie, F-75013 Paris, Francee \\ E-mail: ang.1@cern.ch
}

After the Higgs boson discovery at the LHC in 2012 by the ATLAS and CMS experiments, the measurement of the properties of the Higgs boson became the priority among the experimental particle physics community. The Higgs boson mass and the third family $(t, b$ and $\tau$ ) Yukawa couplings have now been measured at the LHC. The best accuracy on the Higgs boson mass, which is currently $140 \mathrm{MeV}$. In order to measure the Higgs boson properties at high precision, the high energy future collider programs were proposed. In this essay, the $\mathrm{ZH}$ cross-section measurement with "recoil mass" technique, the electron Yukawa coupling, and the Higgs boson self-coupling measurements at FCC will be discussed.

\footnotetext{
*** Particles and Nuclei International Conference - PANIC2021 ***

*** 5 - 10 September, $2021 * * *$

$* * *$ Online $* * *$

${ }^{1}$ On behalf of the FCC Collaboration

${ }^{*}$ Speaker
} 


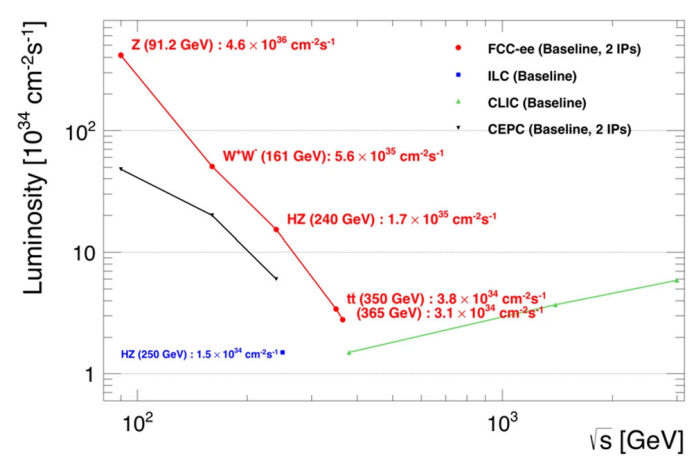

(a) Luminosity as a function of c.o.m for the FCC-ee with two interaction points. The simulated luminosity is shown. Also shown are those estimated for ILC, CLIC and CEPC.

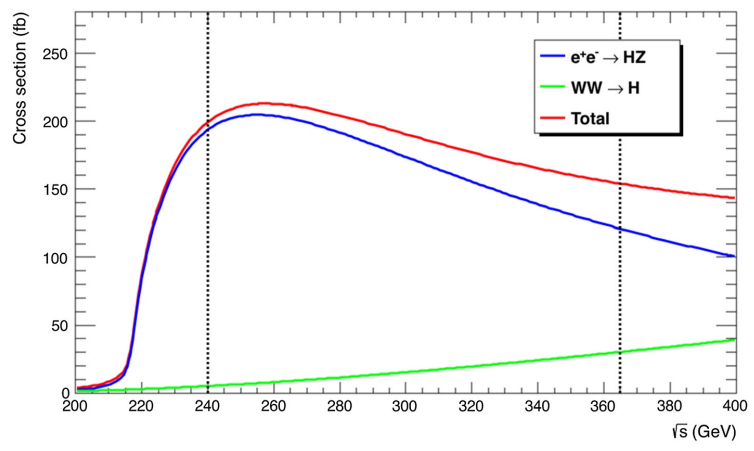

(b) The Higgs boson production cross-section as a function of the c.o.m energy in unpolarised $e^{+} e^{-}$collisions. The vertical dotted lines indicate the c.o.m energies of choice at the FCC-ee for the measurement of the Higgs boson properties.

Figure 1: Luminosity and cross-section at electron-positron colliders Ref. [1]

\section{Future Colliders}

Two types of electron-positron accelerators, linear, and circular colliders, are proposed for future experiments by the particle physics community. For linear colliders, the International Linear Collider (ILC) was proposed to be constructed in Japan while the Compact Linear Collider (CLIC) was proposed by CERN. They are both electron-positron colliders working at different centre-ofmass (c.o.m) energies $(\sqrt{s})$. For circular collider, CERN proposes the Future Circular Collider (FCC) while the Chinese community proposes the Circular Electron-Positron Collider (CEPC). The design of FCC and CEPC are similar. Both of them will first work as an electron-positron machine then upgrade to become hadron colliders. In this document, we will focus on the FCC.

The current strategy for FCC, agreed in 2020, sets an electron-positron Higgs boson factory as the highest priority facility after the Large Hadron Collider (LHC), along with the investigation of the technical and financial feasibility of such a Higgs boson factory, followed by a hadron collider placed in the same tunnel, about $90 \mathrm{~km}$ long. Among the 18 years of preparation, the five-year feasibility study of FCC will begin alongside with the LHC-Run3 in 2021. The next European Strategy Update will be held around 2026 to decide the approval of the project. If approved, the civil engineering, accelerator and detector constructions will begin. The whole FCC integrated program (FCC-INT) is similar to the LEP-LHC program (the Large Electron-Positron collider followed by the LHC in the same tunnel). The first stage of FCC will run as an electron-positron machine (FCC-ee) at different c.o.m energy points for 15 years. Then, there will be a 10 years long shutdown to upgrade the machine to FCC hadron collider (FCC-hh) including the change of magnets and detectors. FCC-hh will then take data for 25 years.

\section{Higgs Measurements at Future Circular Collider}

The FCC-ee will operate at several c.o.m energy points, the $\mathrm{Z}$ pole at $91.2 \mathrm{GeV}$, WW threshold at $161 \mathrm{GeV}, \mathrm{ZH}$ peak at $240 \mathrm{GeV}$ and close to the $t \bar{t}$ threshold at $365 \mathrm{GeV}$, are shown in Fig. 1a. 
FCC-ee will produce $5 \times 10^{12} \mathrm{Z}$ bosons, which is five times more than the number of $\mathrm{Z}$ bosons produced by LEP, $10^{8} \mathrm{WW}$ events at WW threshold. The Higgsstrahlung $(\mathrm{ZH})$ process, $e^{+} e^{-} \rightarrow Z H$, and the WW fusion process, $e^{+} e^{-} \rightarrow H v_{e} \overline{v_{e}}$, are essential for the Higgs boson measurements. Fig. 1b shows the variation of the cross-section of the $\mathrm{ZH}$ and $\mathrm{WW}$ production modes with the c.o.m energy. The cross-section of Higgsstrahlung process is maximal at about $260 \mathrm{GeV}$, but the $\mathrm{ZH}$ event rate is largest at $240 \mathrm{GeV}$. About $10^{6}$ events will be produced with the expected integrated luminosity of $5 \mathrm{ab}^{-1}$. At $365 \mathrm{GeV}$, the machine will produce $1.8 \times 10^{5} \mathrm{ZH}$ and $4.5 \times 10^{4} \mathrm{WW}$-fusion events. Combining the data collected at these two energies will improve the Higgs boson measurements significantly.

ZH cross-section measurement with the Higgs boson "recoil mass" method The progress made with the Higgs boson recoil mass method was reported in Ref. [2]. Only the leptonic decays of $\mathrm{Z}$ boson $\left(Z \rightarrow l^{+} l^{-}\right.$, with $l=e$ or $\mu$ ) are used for the cross-section measurement, as these can be measured very precisely and allow for the $\mathrm{ZH}$ events to be inclusively and efficiently selected independently of the Higgs boson decay mode. This choice is therefore effective towards an almost fully model-independent determination of the HZZ coupling, but the small $\mathrm{Z}$ di-electron and di-muon branching ratios limit the statistical precision. The mass $m_{\text {recoil }}$ recoiling against the lepton pair is determined from total energy-momentum conservation that is shown in Eq. 1 and figuratively in Fig. 2 calculating the difference of the four-vector of c.o.m energy and lepton pair system.

$$
m_{\text {recoil }}^{2}=\left(\sqrt{s}-E_{l \bar{l}}\right)^{2}-p_{l \bar{l}}^{2}=s-2 E_{l \bar{l}} \sqrt{s}+m_{l \bar{l}}^{2}
$$

Since it uses the c.o.m energy, the recoil mass is sensitive to its precise knowledge, which can be

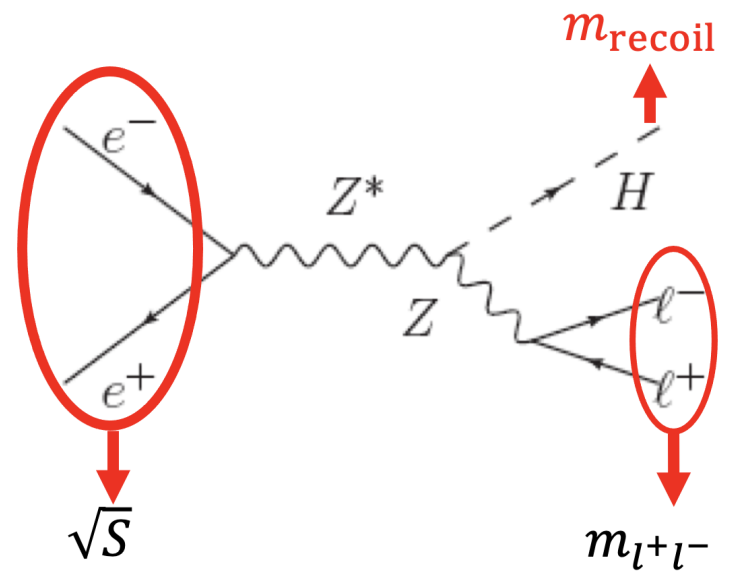

Figure 2: Feynman diagram of Higgsstrahlung process and "Recoil mass", $m_{\text {recoil }}$, calculation.

affected by the beam energy spread (BES) and initial state radiation (ISR). The main backgrounds come from the WW, ZZ and dilepton processes. Fig. 3a shows the $m_{\text {recoil }}$ distribution between 40 and $160 \mathrm{GeV}$, where two peaks are present, one around $125 \mathrm{GeV}$, from the $\mathrm{ZH}$ process, while another is around $91 \mathrm{GeV}$, from the $\mathrm{ZZ}$ process.

Ultimately, the $\sigma_{\mathrm{ZH}}$ and the Higgs boson mass accuracy are expected to achieve $0.5 \%$ and $\mathrm{MeV}$ level respectively [2]. After measuring the ZH cross-section, the HZZ coupling and Higgs boson width can be determined and are expected to achieve per mille precision. 


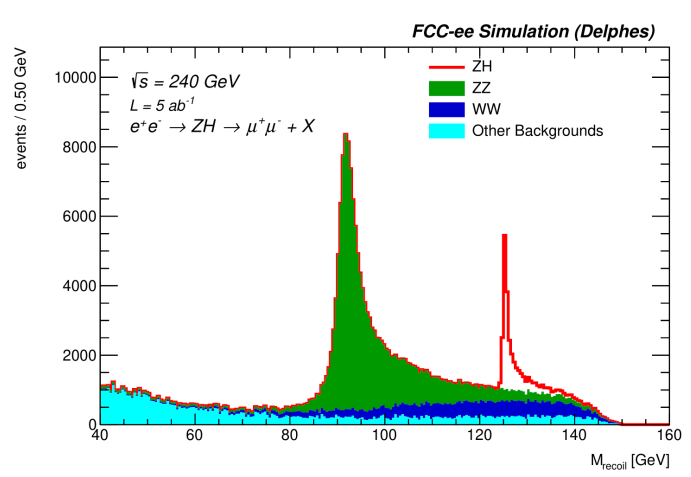

(a) Luminosity as a function of c.o.m for the FCC-ee with two interaction points. The simulated luminosity is shown. Also shown are those estimated for ILC, CLIC and CEPC.

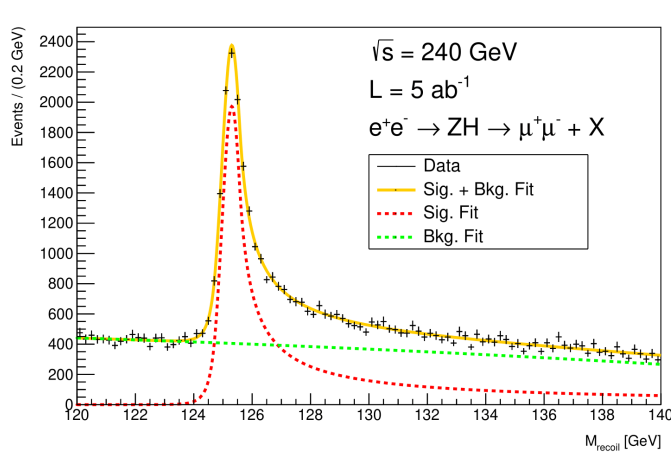

(b) The Higgs boson production cross-section as a function of the c.o.m energy in unpolarised $e^{+} e^{-}$collisions. The vertical dotted lines indicate the c.o.m energies of choice at the FCC-ee for the measurement of the Higgs boson properties.

Figure 3: Recoil mass distribution and fits Ref. [2]

Though the $\mathrm{ZH}$ recoil mass study is still ongoing here we show some preliminary results. We only studied the $\mu^{+} \mu^{-}$final states, applied simple selections, without applying advanced analysis technique, and then determine the signal yield and peak position through a fit to the $m_{\text {recoil }}$ distribution of the selected candidates. Fig. 3b shows an example of the signal plus background fit. So far, we conclude that the statistical analysis yields an Higgs boson mass uncertainty of 6.7 $\mathrm{MeV}$. Inclusion of the systematic uncertainties results into about $8.0 \mathrm{MeV}$ and $1.9 \%$ uncertainties respectively, where the uncertainty from ISR is dominant but conservatively estimated.

Higgs-Electron Yukawa coupling The electron Yukawa coupling measurement was studied in Ref. [3]. After achieving MeV precision on the Higgs boson mass, the electron Yukawa coupling could be measured via the resonant s-channel $e^{+} e^{-} \rightarrow H$ by operating the FCC-ee at $\sqrt{s}=m_{H}$. So far, the Yukawa couplings have been measured only for top $(t)$, bottom $(b)$ quarks and $\tau$ lepton. At the end of the high luminosity LHC (HL-LHC) era, part of the Higgs boson Yukawa couplings to the second family fermions will be probed. But the Yukawa couplings to the first family fermions are difficult to probe since the Higgs boson branching ratio is proportional to the square of the mass of the fermion. The resonant Higgs boson production was considered so far only for a muon collider since the cross-section of $e^{+} e^{-} \rightarrow H$ is about $1.64 f b$ while for muon $\left(\mu^{+} \mu^{-} \rightarrow H\right)$ the cross-section is about $70 \mathrm{pb}$. There are several challenges for this measurement. First, we need a very accurate knowledge of the Higgs mass, which is expected to be achieved with the ZH recoil studies. Second, the ISR and BES need to be known at about the MeV level. And finally, several backgrounds have to be known precisely. But this measurement has many fundamental physics motivations and implications. In particular, the Higgs mechanism for first family of fermions can be probed experimentally.

Trilinear Higgs self-coupling The Higgs boson self-coupling measurement at FCC was studied in Ref. [4] and [5]. The self-coupling can be measured at FCC-ee using the loop-induced modifications to single-Higgs production. If we include the entire FCC-ee dataset, a model-independent precision 
of $42 \%$ can be achieved on $\kappa_{\lambda}$. It will be reduced to $34 \%$ in combination with HL-LHC data, to $21 \%$ with four interaction points (IPs). The Higgs boson self-coupling could be discovered at FCC-ee with four IPs. It will be reduced to $12 \%$ when only $\kappa_{\lambda}$ is allowed to vary and the other parameters are taken at their SM values. The FCC-hh has the potential to reach a precision of 5\% on $\kappa_{\lambda}$ from di-Higgs production in combination with the precise Higgs boson decay branching ratio measurements from the FCC-ee.

\section{Conclusion}

In the Higgs boson measurements at the electron-positron colliders, the "ZH recoil mass" method will improve the uncertainty of $m_{H}$ to a few $\mathrm{MeV}$ level (and the Higgs boson width $\Gamma$ $=4.1 \mathrm{MeV}$ in the $\mathrm{SM}$, could also be measured), the precision on $\sigma_{\mathrm{ZH}}$ to $0.5 \%$, and measure the HZZ coupling as a "candle" for other Higgs boson studies. The electron Yukawa coupling could be probed via the s-channel production at FCC-ee. The trilinear Higgs boson self-coupling could be discovered with four IPs at FCC-ee and will be studied precisely at FCC-hh. Results in this document are preliminary, and updated studies are ongoing.

\section{References}

[1] FCC collaboration, FCC Physics Opportunities: Future Circular Collider Conceptual Design Report Volume 1, Eur. Phys. J. C 79 (2019) 474.

[2] P. Azzurri and et al, A special Higgs challenge: Measuring the mass and production cross section with ultimate precision at FCC-ee, 2106.15438.

[3] D. d'Enterria, A. Poldaru and G. Wojcik, Measuring the electron Yukawa coupling via resonant s-channel Higgs production at FCC-ee, 2107.02686.

[4] M. McCullough, An Indirect Model-Dependent Probe of the Higgs Self-Coupling, Phys. Rev. D 90 (2014) 015001 [1312.3322].

[5] A. Blondel and P. Janot, Future strategies for the discovery and the precise measurement of the Higgs self coupling, 1809.10041. 